\title{
FDTD Optical Simulation for Organic Solar Cells Incorporated with Antireflection Nanostructures
}

\author{
Shigeru Kubota ${ }^{1 *}$, Kensaku Kanomata ${ }^{1}$, \\ Bashir Ahmmad $^{1}$, Jun Mizuno ${ }^{2}$, Fumihiko Hirose ${ }^{1}$ \\ ${ }^{1}$ Graduate School of Science and Engineering, Yamagata University \\ ${ }^{2}$ Research Organization for Nano and Life Innovation, Waseda University \\ ${ }^{\star} k u b o t a @ y z$. yamagata-u.ac.jp
}

Received: December 10, 2019; Accepted: March 31, 2020; Published: April 18, 2020

\begin{abstract}
To improve the performance of organic photovoltaics, we investigate an integrated device design in which the hybrid antireflection structure, composed of a surface moth-eye nanotexture and a multilayer interference film, is applied with a high-refractive-index glass substrate. The moth eye texture with a relatively long period, which is near the bandgap wavelength of organic semiconductors, is used to enhance light absorption. We perform the optical finite-difference time-domain simulation for the integrated device and find the optimal layer configuration of the multilayer interference film to maximize the photocurrent generation. In addition, we compare the absorption spectrum of the integrated device and that of the device with only moth eye coating, and show that the integrated structure is beneficial to realize a high level of absorption relatively uniformly as function of wavelength.
\end{abstract}

Keywords: FDTD, Organic solar cell, Optical simulation, Optimization

\section{Introduction}

Organic photovoltaic (OPV) solar cells, which make use of organic semiconductors as a photoactive layer, are promising as low-cost and flexible power generation devices in the near future [1]. The power conversion efficiency of bulk heterojunction OPVs has been rapidly increasing during the past decade, and has reached more than $10 \%$. However, the efficiency of OPVs is still lower than the conventional Si-based photovoltaics, and it is required to further improve the performance for widespread use. An important factor limiting the conversion efficiency of OPVs is that the active layer thickness should be very low $(\sim 100 \mathrm{~nm})$ to permit efficient carrier extraction. The use of such thin active layer acts to prevent sufficient absorption of incident light and restrict photocurrent generation. Therefore, it is a quite important 


\section{Journal of Advanced Simulation in Science and Engineering}

issue to develop a high-performance antireflection method to trap light in the thin active layer.

In our previously published study [2], we have proposed an integrated device design of OPVs to attain broadband photocurrent enhancement. In this device design, a hybrid antireflection structure (ARS), which consists of 3-dimensional nanotexture and interference multilayer, is integrated with a glass substrate having a refractive index higher than a normal value. It has been shown that the hybrid ARS can effectively suppress the reflection occurring at the front side of the glass substrate, while the use of the high-refractive-index glass can decrease the reflection occurring at the back side of the substrate. Therefore, the combination of them serves to significantly decrease the overall level of reflection for the whole device. For the nanotexture contained in the hybrid ARS, the moth eye texture (i.e., a structure in which the nanometer-size cones are periodically arranged) has been used. The period of the moth eye pattern has been set to be $250 \mathrm{~nm}$, which is shorter than the wavelength of sunlight ( $>$ $300 \mathrm{~nm}$ ). The use of the short moth eye period is based on the results of past studies that suggest that, when the length of period is sufficiently short, the effective refractive index changes gradually within the moth eye array and the reflection occurring due to a discontinuous change in the index value can be significantly suppressed [3].

On the other hand, our another previous study [4], which has analyzed the optical property of the moth eye attached to the front surface of an OPV device, has shown that a longer period of moth eye is beneficial to enhance the performance. This study has applied the finite-difference time-domain (FDTD) simulation to optimize the geometric parameters of moth eye, and has found the optimized period of $592 \mathrm{~nm}$, which is relatively near the bandgap wavelength of organic semiconductors $(653 \mathrm{~nm})$. The incident light near this wavelength is known to be generally difficult to be absorbed in the active layer. But, by applying the longer period of moth eye, the optical wave with the wavelength around the period is significantly bent by diffraction, strengthening the intensities of electric field and absorption within the thin active layer [4]. Adequately selecting the longer moth eye period would be effective in improving the light trapping performance.

Therefore, in this study, we apply the moth eye texture with the longer period to the integrated OPV device [2], and explore the resultant change in the performance. We conduct optimization of the thicknesses of interference multilayer within the hybrid ARS, and elucidate the layer configuration to achieve the maximum photocurrent level. Additionally, we compare the absorption spectrum between the integrated device and the device with only the moth eye coating to clarify the change in optical characteristics depending on the antireflection configuration. 


\section{Methods}

\subsection{OPV device model}

We performed the FDTD simulation for the OPV devices shown in Fig. 1. The layer configuration of the OPV cell is described as glass substrate/ITO $(150 \mathrm{~nm}) / \mathrm{MoO}_{3}(7 \mathrm{~nm}) /$ poly(3-hexylthiophene) (P3HT):[6,6]-phenyl-C 61 -butyric acid methyl ester (PCBM) (100 $\mathrm{nm}) / \mathrm{Al}(100 \mathrm{~nm})$ [4]. Figure 1a shows the reference OPV cell (i.e., the device without ARS), and Fig. $1 \mathrm{~b}$ shows the device with the moth eye antireflection coatings. In Figs. 1a and 1b, the glass substrate has a normal refractive index (normal $n$ ) of 1.5. Figure 1c shows the integrated OPV device in which the hybrid ARS is used in combination with a high-refractive-index (high $n$ ) glass substrate with the refractive index of 1.75 [2]. The hybrid ARS consists of the moth eye array and the interference film made of $\mathrm{Al}_{2} \mathrm{O}_{3}$ and $\mathrm{MgF}_{2}$ layers (with the thicknesses $d_{1}$ and $d_{2}$, respectively) [2]. The materials of $\mathrm{Al}_{2} \mathrm{O}_{3}$ and $\mathrm{MgF}_{2}$ are selected because they are typically used for antireflection coating. Throughout the current study, the moth eye array is assumed to have the period of $592 \mathrm{~nm}$ and the height of $600 \mathrm{~nm}$, which

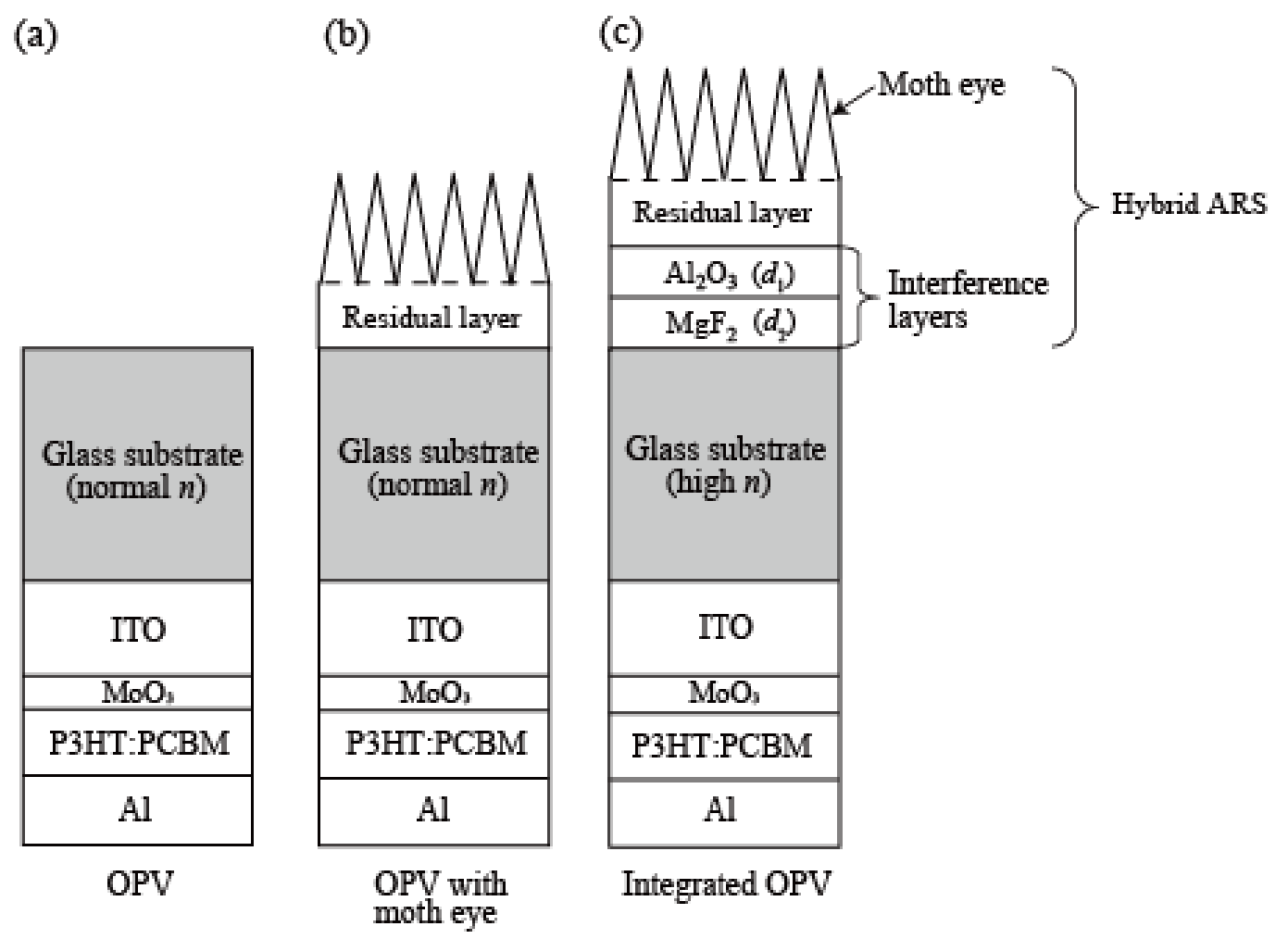

Figure 1: OPV devices used in the current simulation. (a) Structure of a reference OPV cell. (b and c) Structures of the OPV cell with the moth eye coating (b) and with the integrated design (c). In (c), $d_{1}$ and $d_{2}$ represent the thicknesses of the $\mathrm{Al}_{2} \mathrm{O}_{3}$ and $\mathrm{MgF}_{2}$ layers, respectively. 


\section{Journal of Advanced Simulation in Science and Engineering}

have been obtained in the above-mentioned study [4]. This value of period is significantly longer than the value of $250 \mathrm{~nm}$, which is used in the previous study on the integrated device [2], as mentioned above. The refractive index of the material of the moth eye structure is set as 1.5 [4]. This is a typical value for the organic polymers used for the nanoimprint lithography, which is a widely used method for fabricating nanostructures. It is assumed that there is a residual layer (a $500 \mathrm{~nm}$-thick polymer film) adjacent to the moth eye, which is generally present when the nanostructure is fabricated with nanoimprint lithography.

\subsection{FDTD simulation with envelope algorithm}

In the FDTD simulation for the cases where the light goes through a glass substrate, the interference of light reflecting at the front and back sides of the glass substrate elicits an optical response oscillating significantly as function of wavelength [4]. This kind of oscillatory response is considered artificial in the sense that it does not exist in the real device. This is because, within the glass substrate much thicker than the wavelength of incident light, the optical coherence disappears and the addition of irradiances occurs between multiple optical waves. To remove the artificial oscillation in the FDTD response and accurately predict the performance of the examined device, we applied the envelope algorithm $[4,5]$. This algorithm can be summarized as follows. As shown in Fig. 2, we define $r(\lambda)$ as an arbitrary oscillatory response (e.g., absorbance), obtained with the FDTD simulation, as function of wavelength $\lambda$. We construct the upper and lower envelopes of function $r(\lambda)\left(f_{+}(\lambda)\right.$ and $f_{-}(\lambda)$, respectively) by employing the spline interpolation of the local peaks and troughs of this function. Then, by taking the average between both the two envelopes, we can obtain the

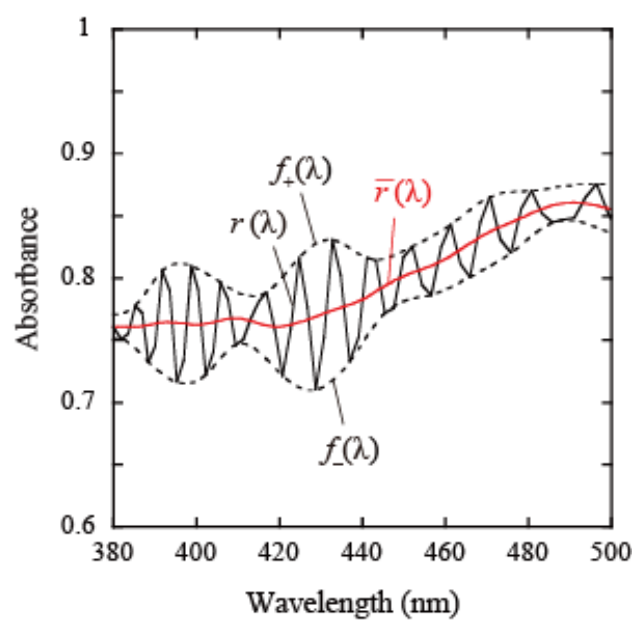

Figure 2: An example of applying the envelope algorithm to the spectrum of absorbance in the active layer obtained by FDTD simulation. 


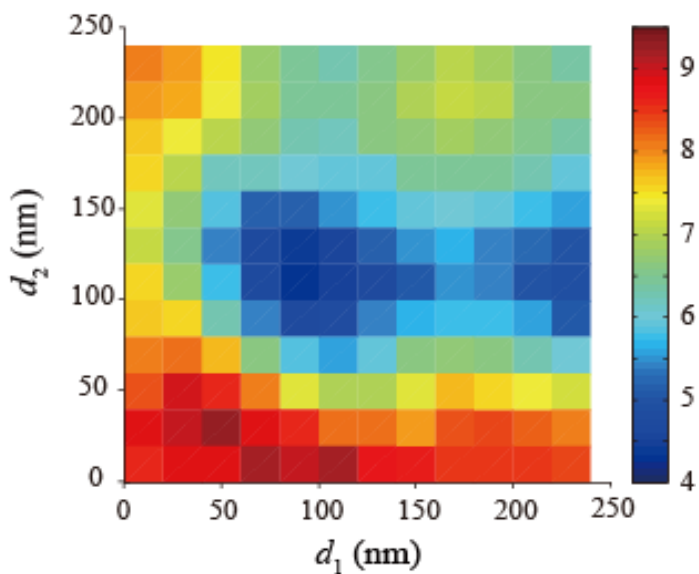

Figure 3: Relative changes (in percentage) in $J_{S C}$ of the integrated OPV cell as function of $d_{1}$ and $d_{2}$.

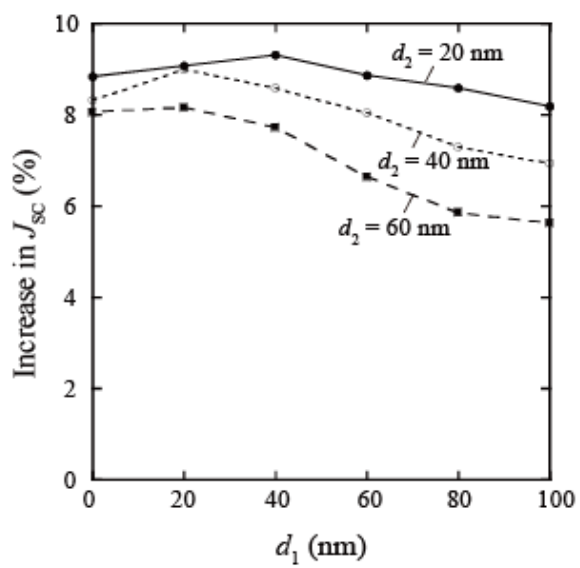

Figure 4: Relative changes in $J_{S C}$ of the integrated OPV cell as function of $d_{1} .\left[d_{2}=20 \mathrm{~nm}\right.$ (solid), $40 \mathrm{~nm}$ (dotted), or $60 \mathrm{~nm}$ (dashed).]

optical response $\left(\bar{r}(\lambda)=\left(f_{+}(\lambda)+f_{-}(\lambda)\right) / 2\right)$ in which the artificial interference effect has been eliminated. We applied the envelope algorithm to obtain the wavelength dependence of the irradiance absorbed in the active layer, and calculated the magnitude of short-circuit current density $\left(J_{S C}\right)$ based on the integral of the absorbed irradiance over wavelength [4]. $J_{S C}$ is defined as the current level obtained when the electrodes of solar cells are short circuited, and reflects the overall level of light absorption in the active layer.

\section{Results}

We investigated the change in $J_{S C}$ depending on the thicknesses of interference multilayer of the integrated device (Fig. 1c) to find its optimal configuration. The relative increase in $J_{S C}$, as compared to the value for the reference cell, is shown as function of $d_{1}$ and $d_{2}$ in Fig. 3. Since the figure suggests that the photocurrent level is relatively high in the lower-left area (i.e., the area where $d_{1}$ and $d_{2}$ are smaller), the change in $J_{S C}$ corresponding to the smaller values of $d_{1}$ and $d_{2}$ is clarified in Fig. 4 . The optimal layer configuration was found to be $d_{1}=40 \mathrm{~nm}$ and $d_{2}=20 \mathrm{~nm}$ at which the increase rate of $J_{S C}$ is $9.3 \%$. This increase rate is significantly greater than the value of $5.7 \%$, which is obtained when the moth eye period is smaller $(250 \mathrm{~nm})$ as in the case of the previous study [2], and is also greater than the value of $8.4 \%$ obtained when only the moth eye coating is applied as in Fig. $1 \mathrm{~b}$. The result here clarifies the validity of selecting the longer period of moth eye texture for the photocurrent enhancement. 


\section{Journal of Advanced Simulation in Science and Engineering}

Furthermore, we compared the absorption spectra corresponding to the three device configurations in Fig. 1. Here, the optimized values of $d_{1}$ and $d_{2}$ were used for the thicknesses of interference multilayer of the integrated device. Figures 5 and 6 show the comparison of the spectra of the absorbed energy in the active layer for the three cases (Fig. 5) and the relative difference between them (Fig. 6). The result of Fig. 6 indicates that the absorbed energy tends to be significantly enhanced by applying either the moth eye or the integrated design, particularly in the longer wavelength around the moth eye period (592 nm) [4]. In addition, Fig. 6 clarifies that the increase level of absorption is more uniform with the integrated device design. In fact, the range of the increase in the absorbed energy is $[-0.02,0.2]\left(\right.$ in $\left.\times 10^{9} \mathrm{Wm}^{-2} \mathrm{~m}^{-1}\right)$ for the case with moth eye (Fig. 6, black line), which is narrowed down to $[0,0.16]$ for the case with the integrated design (Fig. 6, red line). It also should be noted that there is a range of wavelength ( $\sim 420 \mathrm{~nm})$ at which the absorption level is decreased by applying the moth eye (Fig. 6, black line), whereas the absorption level is enhanced for all the wavelength range by applying the integrated design (Fig. 6, red line). These results suggest that the integrated device design tends to make use of a wide wavelength range of incident waves effectively to improve the overall level of absorption.

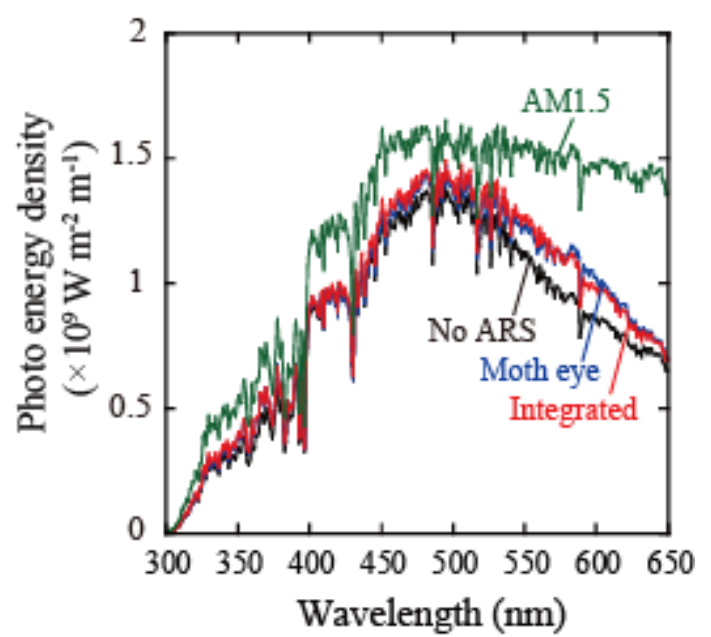

Figure 5: Spectra of the energy absorbed in the active layer. The black line shows the case without ARS (Fig. 1a), whereas the blue and red lines show the cases with the moth eye (Fig. 1b) and integrated design (Fig. 1c), respectively. The green line represents the AM1.5 solar irradiance spectrum, for the comparison.

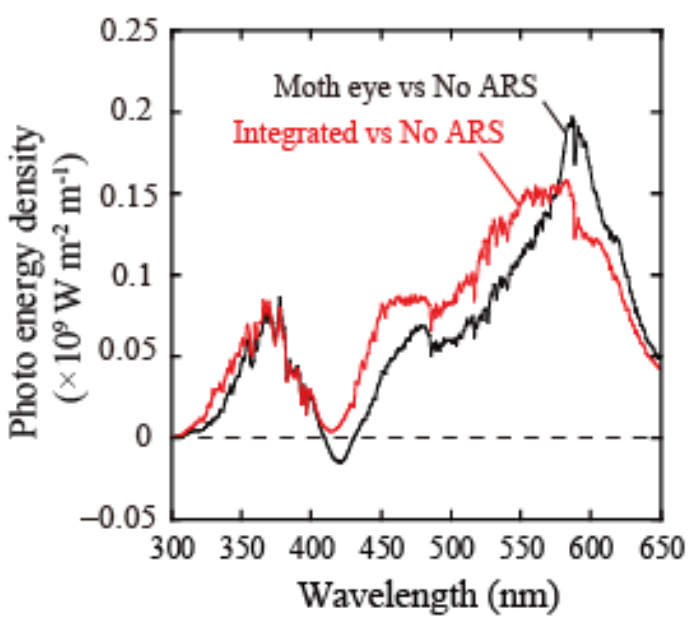

Figure 6: The black line shows the difference in the energy absorbed in the active layer between the cases of applying the moth eye (Fig. 1b) and not applying ARS (Fig. 1a). The red line is the difference between the cases of using the integrated design (Fig. 1c) and not using ARS (Fig. 1a). 


\section{Journal of Advanced Simulation in Science and Engineering}

\section{Conclusion}

In this study, the FDTD simulation has been applied to obtain the optimized layer configuration of the interference multilayer for the integrated OPV device [2]. It has been shown that by using the longer moth eye period, which is relatively near the wavelength corresponding to the bandgap of organic semiconductors, the photocurrent level can be significantly increased. This result implies that it would be important to adequately decide the design target wavelength for the moth eye texture to fully bring out its light trapping ability [4]. In addition, it has been clarified that the absorption in the active layer is enhanced relatively uniformly in broadband wavelength by applying the integrated design, which plays a role in improving solar cell performance.

\section{Acknowledgement}

This study was partially supported by KAKENHI (17K05001) from the Japanese government.

\section{References}

[1] F. C. Krebs, N. Espinosa, M. Hösel, R. R. Sondergaard, M. Jorgensen: 25th Anniversary Article: Rise to power-OPV-based solar parks, Advanced Materials, volume:26 (2014), $29-39$.

[2] S. Kubota, Y. Harada, T. Sudo, K. Kanomata, B. Ahmmad, J. Mizuno, F. Hirose: An integrated antireflection design using nanotexture and high-refractive-index glass for organic photovoltaics, Journal of Coatings Technology and Research, volume:14 (2017), $1209-1224$.

[3] A. Deinega, I. Valuev, B. Potapkin, Y. Lozovik: Minimizing light reflection from dielectric textured surfaces, Journal of the Optical Society of America A, volume:28 (2011), $770-777$.

[4] S. Kubota, K. Kanomata, B. Ahmmad, J. Mizuno, F. Hirose: Optimized design of moth eye antireflection structure for organic photovoltaics, Journal of Coatings Technology and Research, volume:13 (2016), 201-210.

[5] S. Kubota, K. Kanomata, B. Ahmmad, J. Mizuno, F. Hirose: FDTD analysis for light passing through glass substrate and its application to organic photovoltaics with moth eye antireflection coating, Journal of Photopolymer Science and Technology, volume:29 (2016), 209-214. 